\title{
Cardiac contraction-induced GLUT4 translocation requires dual signaling input
}

Citation for published version (APA):

Luiken, J. J. F. P., Glatz, J. F. C., \& Neumann, D. (2015). Cardiac contraction-induced GLUT4

translocation requires dual signaling input. Trends in Endocrinology and Metabolism, 26(8), 404-410. https://doi.org/10.1016/j.tem.2015.06.002

Document status and date:

Published: 01/08/2015

DOI:

10.1016/j.tem.2015.06.002

Document Version:

Publisher's PDF, also known as Version of record

Document license:

Taverne

\section{Please check the document version of this publication:}

- A submitted manuscript is the version of the article upon submission and before peer-review. There can be important differences between the submitted version and the official published version of record.

People interested in the research are advised to contact the author for the final version of the publication, or visit the DOI to the publisher's website.

- The final author version and the galley proof are versions of the publication after peer review.

- The final published version features the final layout of the paper including the volume, issue and page numbers.

Link to publication

\footnotetext{
General rights rights.

- You may freely distribute the URL identifying the publication in the public portal. please follow below link for the End User Agreement:

www.umlib.nl/taverne-license

Take down policy

If you believe that this document breaches copyright please contact us at:

repository@maastrichtuniversity.nl

providing details and we will investigate your claim.
}

Copyright and moral rights for the publications made accessible in the public portal are retained by the authors and/or other copyright owners and it is a condition of accessing publications that users recognise and abide by the legal requirements associated with these

- Users may download and print one copy of any publication from the public portal for the purpose of private study or research.

- You may not further distribute the material or use it for any profit-making activity or commercial gain

If the publication is distributed under the terms of Article $25 \mathrm{fa}$ of the Dutch Copyright Act, indicated by the "Taverne" license above, 


\title{
Cardiac contraction-induced GLUT4 translocation requires dual signaling input
}

\author{
Joost J.F.P. Luiken, Jan F.C. Glatz, and Dietbert Neumann
}

Department of Molecular Genetics, Cardiovascular Research Institute Maastricht (CARIM), Maastricht University, NL-6200 Maastricht MD, the Netherlands

\begin{abstract}
Contraction-induced translocation of glucose transporter type-4 (GLUT4) to the sarcolemma is essential to stimulate cardiac glucose uptake during increased energy demand. As such, this process is a target for therapeutic strategies aiming at increasing glucose uptake in insulin-resistant and/or diabetic hearts. AMP-activated protein kinase (AMPK) and its upstream kinases form part of a signaling axis essential for contraction-induced GLUT4 translocation. Recently, activation of protein kinase-D1 (PKD1) was also shown to be as obligatory for contraction-induced GLUT4 translocation in cardiac muscle. However, contraction-induced PKD1 activation in this context occurs independently from AMPK signaling, suggesting that contraction-induced GLUT4 translocation requires the input of two separate signaling pathways. Necessity for dual input would more tightly couple GLUT4 translocation to stimuli that are inherent to cardiac contraction.
\end{abstract}

Importance of GLUT4 translocation in cardiac contraction-induced glucose uptake

The rate-limiting step in glucose utilization in muscle cells is firmly established to be the trans-sarcolemmal passage of glucose via one of the muscle- and/or heart-specific GLUTs [1,2]. The heart expresses mainly GLUT1 and GLUT4 (see Glossary), and cardiac GLUT4 expression is approximately four times higher than that of GLUT1 $[3,4]$. Whereas GLUT1 is mainly present constitutively at the sarcolemma and is involved in basal glucose uptake, GLUT4 resides predominantly in intracellular storage compartments, from where it translocates to the sarcolemma to increase glucose uptake in response to insulin, contraction, or various pharmacological stimuli [5].

Given that contraction stimulation can recruit GLUT4 to the sarcolemma independently of the insulin-signaling axis, both in muscle and heart [6,7], pharmacological inhibition of the insulin-signaling pathway fails to inhibit contractioninduced GLUT4 translocation. Based on its independence from the insulin-signaling axis, contraction-induced GLUT4 translocation is of considerable therapeutic interest,

Corresponding author: Luiken, J.J.F.P. (j.luiken@maastrichtuniversity.nl). Keywords: cardiac contraction; glucose uptake; signaling pathway; diabetes; heart. 1043-2760/

(c) 2015 Elsevier Ltd. All rights reserved. http://dx.doi.org/10.1016/j.tem.2015.06.002 because this pathway presents an alternative route to upregulate glucose uptake in the diabetic heart and muscle, thereby circumventing the blockade in insulin-stimulated GLUT4 translocation seen in diabetes [8].

In this opinion article, we discuss established factors and signaling pathways involved in contraction-induced

\section{Glossary}

AMP-activated protein kinase (AMPK):: a heterotrimeric complex comprising a catalytic $\alpha$ subunit $(\alpha 1, \alpha 2$; harboring the kinase domain), a regulatory $\beta$ subunit $(\beta 1, \beta 2$; carrying the carbohydrate-binding module for attachment at glycogen granules), and a regulatory $\gamma$ subunit $(\gamma 1, \gamma 2, \gamma 3$; containing the AMP binding domains). In addition to stress and ischemia, contraction is one of the major stimuli inducing AMPK activation.

Calcium/calmodulin-dependent protein kinase kinase $\beta$ (СаМКК $\beta)::$ a rise in cytoplasmic $\mathrm{Ca}^{2+}$ stimulates a canonical $\mathrm{Ca}^{2+}$ signaling pathway comprising CaMKK $\beta$ and $\mathrm{Ca}^{2+} /$ calmodulin-activated kinase-I (CaMKI). CaMKK $\beta$ is an alternative upstream kinase of AMPK. Upon contraction, CaMKK $\beta$ is likely involved in AMPK activation in skeletal muscle [62], but not in heart [16]. Death-associated protein kinase (DAPK):: mainly known as tumor suppressor gene, whose expression is greatly reduced in various human malignancies. DAPK is activated by ROS and has recently been established as a PKD1activating kinase.

Glucose transporter-4 (GLUT4):: belongs to a group of glucose transporters that facilitates transport of glucose across the plasma membrane. GLUT4 is localized at the plasma membrane, and in intracellular (endosomal) compartments, between which it constantly recycles. An increase in GLUT4-mediated glucose uptake is achieved via increased GLUT4 exocytosis from the endosomes and/or decreased GLUT4 endocytosis and/or internalization, resulting in net GLUT4 translocation. The most important physiological stimuli inducing GLUT4 translocation are insulin and increased muscular activity. Insulin-stimulated GLUT4 translocation is mediated via autophosphorylation of the insulin receptor that initiates a canonical signaling pathway involving subsequent activation of insulin receptor-substrate $1 / 2$, phosphatidyl-inositol-3 kinase, and Akt.

Liver kinase-B1 (LKB1):: a tumor suppressor kinase (mutated or deleted in Peutz-Jeghers syndrome) and a major upstream kinase of AMPK. It also directly activates a family of AMPK-related kinases, such as sucrose nonfermenting AMPK-related kinase (SNARK).

Protein kinase C (PKC):: the PKCs are a family of Ser/Thr kinases with a distinctive activation mechanism involving membrane attachment and subsequent release of a pseudosubstrate region from the catalytic domain. PKCs are classified in three subfamilies that differ in their responsiveness towards the second messengers $\mathrm{Ca}^{2+}$ and DAG (or phorbol esters: cell-permeable DAG analogs). Conventional PKCs are activated by both messengers, novel PKCs only by DAG and atypical PKCs are nonresponsive to either messenger. Protein kinase-D1 (PKD1):: also known as PKC $\mu$, PKD1 is a cytosolic serinethreonine kinase that is closely related to PKC. PKD1 is activated by phorbol esters, the classical PKC activators. However, the catalytic domain of PKD is more closely related to that of the CaMKs, and displays relatively little homology to catalytic domains of the PKC family. In contrast to PKCs, PKD has an additional pleckstrin homology domain and a putative transmembrane sequence, and lacks a pseudosubstrate region, thereby justifying its classification as a novel kinase family.

Transforming growth factor- $\beta$-activated protein kinase-1 (TAK1):: participates in cell responses to environmental stresses and can activate AMPK. 
GLUT4 translocation, and present recent findings showing that the serine/threonine kinase PKD1 is a novel player, inducing a separate pathway that cooperates with previously recognized mechanisms to control contraction-induced GLUT4 translocation. We propose the existence of a dual signaling input system and discuss its putative pathophysiological implications.

\section{AMPK and contraction-induced GLUT4 translocation}

Research in the area of contraction-induced GLUT4 translocation was boosted by the discovery of AMPK involvement [9]. This kinase is activated by increased intracellular AMP concentrations as a consequence of increased ATP utilization, for example upon contraction. AMP directly binds to AMPK, thereby allosterically stimulating simultaneously its phosphorylation and activation by upstream kinases, increasing its enzymatic activity, and decreasing the rate of dephosphorylation. AMPK has been found to activate numerous catabolic pathways while inhibiting anabolic processes, thereby acting as an energy switch to favor ATP production [10]. A role for AMPK in exercise-induced glucose uptake in skeletal muscle has been firmly established [11]. In a nutshell, evidence for AMPK-dependent stimulation of glucose uptake in skeletal muscle is compelling, although loss of AMPK function did not prevent contraction-induced glucose uptake [12].

Compared with muscle, less is known about the role of AMPK in contraction-induced glucose uptake in the heart. In particular, knockout (KO) or transgenic AMPK mouse models have rarely been used to investigate contraction-induced glucose uptake. Assessment of a contraction-mimetic stimulation protocol in cardiomyocytes from mice overexpressing a kinase-dead $\alpha 2$ mutant, and studies in AMPK $\alpha 2-\mathrm{KO}$ mice, have demonstrated that AMPK is also essential for contraction-induced glucose uptake in the heart [13]. However, as we discuss below, the strict requirement of AMPK does not preclude other factors from contributing to exercise-induced GLUT4 translocation.

\section{Factors upstream of AMPK impacting contraction- induced GLUT4 translocation}

The upstream kinases involved in AMPK-mediated GLUT4 translocation have been a topic of intense studies over the past few years. Three different kinases, liver kinase B1 (LKB1), calcium/calmodulin-dependent protein kinase kinase- $\beta(\mathrm{CaMKK} \beta)$, and transforming growth factor $\beta$-activated kinase (TAK1), are capable of activating AMPK by directly phosphorylating amino acid T172 on the $\alpha$-subunit [10]. Among these kinases, the role of LKB1 in contraction-induced glucose uptake has been more extensively studied. Muscle-specific LKB1-deficient mice have demonstrated a major involvement of this kinase in this process in skeletal muscle [14]. Additionally, cardio-specific LKB1-KO mice indicate a major role of LKB1 in AMPK activation in response to ischemia [15]. In cardiomyocytes from LKB1-KO mice, glucose uptake stimulation by a contraction mimetic was entirely abolished [13], whereas in rat cardiomyocytes, pharmacological inhibition of CaMKK $\beta$ by the potent CaMKK inhibitor STO-609, had no effect [16]. Thus, in this experimental setting, LKB1 but not CaMKK $\beta$ is required for contraction-induced glucose uptake via AMPK activation. With respect to TAK1, there are no studies so far on its role in contraction-induced glucose uptake.

Hence, at present, LKB1 appears to be the sole AMPK upstream kinase with an established role in cardiac contraction-induced glucose uptake. Given that LKB1 is constitutively active upon formation of a heterotrimeric complex with its natural binding partners, mouse protein 25 (Mo25) and the STE-20-related adaptor protein (STRAD) [17,18], and that AMPK is allosterically regulated by adenine nucleotides, the LKB1 complex appears to have a permissive rather than regulatory role in contraction-induced GLUT4 translocation.

\section{Factors downstream of AMPK impacting contraction- induced GLUT4 translocation}

For identification of downstream AMPK signaling in contraction-induced GLUT4 translocation, lessons can be learned from studying the signaling step in insulin-induced GLUT4 translocation downstream of Akt. This is known to be mediated by phosphorylation of TBC1D4, a member of the tre-2/USP6, BUB2, cdc16 domain-containing family of Rab-GTPases [19,20]. TBC1D4, also known as Akt substrate of $160 \mathrm{kDa}$ (AS160), is an inhibitor of the function of Rab proteins, by keeping them in an inactive GDP-bound state, thereby preventing the GTP-GDP cycling that is necessary to drive GLUT4 translocation. TBC1D4, as established in adipocytes, is phosphorylated on multiple sites by insulin treatment, resulting in binding to the adaptor protein 14-3-3, inactivation of RabGAP activity, and dissociation from the intracellular GLUT4 storage compartment [21,22]. Hence, insulin-induced TBC1D4 phosphorylation relieves the brake on GLUT4 translocation. Originally, it was thought that TBC1D4 would also be phosphorylated by AMPK for contractionstimulation of GLUT4 translocation [23]. Yet, recent studies in skeletal muscle pointed towards TBC1D1, another member of the TBC1 Rab-GTPase family, as being responsible for regulating GLUT4 translocation directly downstream of AMPK [24,25].

More than 70 Rab proteins have been identified so far, and the different members are dedicated to specific trafficking processes [26]. The different TBC members appear to inhibit the activity of a distinct set of Rabs, and this also would be expected to apply for TBC1D1 and TBC1D4 [27]. Whereas TBC1D4 specifically inhibits Rab8A, Rab10, and Rab14 [28,29], the in vitro Rab GAP specificity of TBC1D1 still needs to be determined. In agreement with this, rab8A and Rab14 appear to mediate insulin-induced GLUT4 translocation in muscle cells [28]. It has not yet been assessed whether these or different Rabs are involved in contraction-induced GLUT4 translocation in muscle and heart.

\section{Requirement for PKD1 in contraction-induced GLUT4 translocation}

In addition to AMP, which leads to AMPK activation, other second messengers become elevated during contraction, such as intracellular $\mathrm{Ca}^{2+}$ and diacylglycerol (DAG) 
[30]. Both second messengers are known activators of members of the conventional and novel protein kinase $\mathrm{C}$ (PKC) subfamilies, as well as the closely related PKD family, with its founding member PKD1. Moreover, the classical PKC activators phorbol esters (cell-permeable DAG analogs) additionally activate PKD1.

$\mathrm{PKD} 1$ is involved in many extracellular receptor-mediated signal transduction pathways that function in several fundamental cellular processes, such as cell proliferation and differentiation, membrane trafficking, immune responses, inflammation, angiogenesis, and cancer [31]. Additionally, PKD1 regulates the fission of transport vesicles from the trans-Golgi network to translocate to the cell surface [32,33]. PKD1 has N-terminal tandem cysteinerich $\mathrm{C} 1 \mathrm{~A} / \mathrm{C} 1 \mathrm{~B}$ domains for binding DAG/phorbol esters and a pleckstrin homology domain for protein-protein interactions [31,33].

Several lines of evidence suggest a crucial role for PKD1 in contraction-induced glucose uptake. Pharmacological PKC inhibitors, which also inhibit PKD1 [34,35], as well as RNA silencing of PKD1 were shown to completely abolish contraction-induced GLUT4 translocation and glucose uptake in cardiomyocytes [34]. Similarly, in cardiomyocytes from cardio-specific PKD1-KO mice, contractioninduced glucose uptake was entirely lost [34]. Furthermore, PKD1 overexpression in vitro (using adenovirally mediated transduction in cardiomyocytes [36]), and in vivo (using transgenic mice overexpressing constitutively active cardiac PKD1 [37]) was shown to elevate basal and contraction-induced glucose uptake.

In the heart, one-third of PKD1 appears to be membrane bound [35]. PKD1 associates with the sarcolemma, mitochondria, and the trans-Golgi network [31,33]. The presence of PKD1 within the trans-Golgi network is particularly interesting, because PKD1 activation at the Golgi complex is involved in the formation and budding of vesicles bringing cargo to the plasma membrane [33]. Considering the connection of the trans-Golgi network to the recycling endosomes via an extended network of membranes [38], it is plausible that the recycling endosomes might also harbor PKD1, and endosomal PKD1 might carry out a role analogous to that of Golgi-localized PKD1, that is, the budding of GLUT4-containing vesicles for translocation to the sarcolemma.

\section{Upstream factors activating PKD1}

In cardiomyocytes, PKD1 is activated upon contraction stimulation at levels comparable to those seen in activation by phorbol esters (classical PKC and/or PKD activators) [35]. However, the mechanism of PKD1 activation by contraction is different from phorbol ester-induced activation. Namely, in contrast to the wellestablished phorbol ester-induced transphosphorylation of PKD1 at Ser744 and Ser748 within the activation loop by novel PKCs (nPKCs) and the accompanying membrane attachment, contraction induces neither Ser744/ Ser748 transphosphorylation nor membrane attachment $[35,39]$. Yet, both contraction and phorbol esters similarly induce autophosphorylation at Ser916. Hence, there is a unique PKD1 activation mechanism upon contraction stimulation, suggesting the existence of an upstream pathway different from phorbol ester-elicited PKD1 activation.

PKD1 is activated during periods of oxidative stress by reactive oxygen species (ROS) [33], a well-known by-product of exercise and/or increased muscle contraction. ROSinduced PKD1 activation is mediated by nonreceptor tyrosine kinases, such as c-Abl and Src, which phosphorylate PKD1 at Tyr463 at the pleckstrin homology domain. This then allows PKC $\delta$ to bind and transphosphorylate PKD1 at Ser744/748 [33]. Given that this pathway results in phosphorylation of PKD1 at Ser-744 and at Ser-748 in the activation loop, similar to the $\mathrm{nPKC}$ pathway, it is unlikely to participate in the phosphorylation and/or activation of PKD1 during contraction stimulation in cardiomyocytes. Indeed, a different ROS-activated kinase, namely the death-activated kinase (DAPK), has been reported to bind to and activate PKD1 [40]. Immunoprecipitation experiments in cardiomyocytes indicated that DAPK dramatically increases its binding to PKD1 upon contraction stimulation, and DAPK silencing resulted in loss of both contraction-induced PKD1 autophosphorylation and GLUT4 translocation [34]. Thus, the DAPK-PKD1 complex participates in contraction-induced GLUT4 translocation. Recently, a DAPK-PKD1 signaling axis was found to mediate autophagy via initiation of budding of autophagosomal membranes from the endoplasmic reticulum [41]. These data are in line with the presently envisaged role of DAPK in PKD1-mediated budding of GLUT4-containing vesicles from the endosomal compartment [34]. Hence, the DAPK-PKD1 axis might have a more general role in vesicular budding. It is not known via which mechanism DAPK becomes activated by contraction. Perhaps analogous to AMPK activation by LKB, DAPK is constitutively active, but only displays kinase activity towards PKD1 upon the onset of contraction (via a hypothetical ROS-induced conformational change within PKD1).

\section{Downstream of PKD1}

How would PKD1 signal to endosomes to initiate budding of GLUT4-containing vesicles? There are more than 1000 proteins with a PKD consensus phosphorylation motif. Among these are several Golgi-associated proteins that function in vesicle-mediated transport between the Golgi and the plasma membrane [42]. Two of these proteins are lipid kinases, namely DAG kinase and phosphatidylinositol-4 kinase-III $\beta$ (PI4KB), which generate phosphatidic acid and phosphatidylinositol-4-phosphate (PI4P), respectively. Both lipid species have bilayer-destabilizing properties favoring the formation of curvature into membranes. Of these proteins, PI4KB is a physiological PKD1 substrate involved in the Golgi-to-plasma membrane transport of secretory proteins [32]. Another interesting candidate protein is $\mathrm{C}$ terminus-binding protein 3/brefeldin-A adenosine diphosphate-ribosylated substrate (CtBP3/BARS), which functions as an inducer of fission of vesicles from the trans-Golgi network via an as yet unknown mechanism [43]. It is plausible that these proteins are additionally present in the endosomal compartment, given the filamentous connections between Golgi and endosomes [38]. Moreover, it is reasonable to 


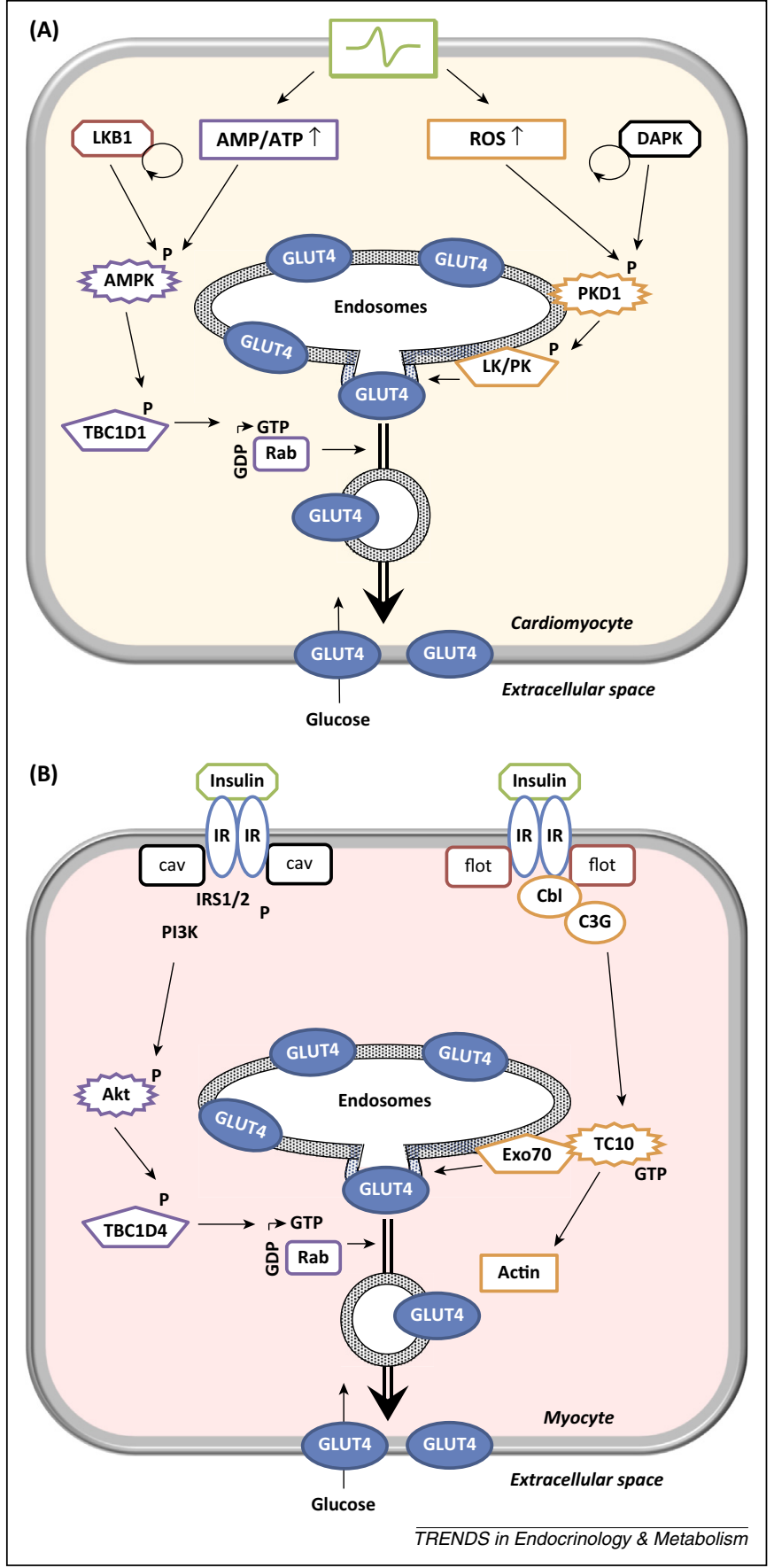

Figure 1. Glucose transporter-4 (GLUT4) translocation modes in cardiac and skeletal muscles. (A) Contraction-induced GLUT4 translocation in myocytes. Contraction simultaneously increases intracellular levels of AMP and reactive oxygen species (ROS). Elevated AMP stimulates the activation of AMP-activated protein kinase (AMPK) by liver kinase-B1 (LKB1) (or alternative upstream kinases). Active AMPK subsequently phosphorylates TBC1D1, resulting in activation of Rabs and enhanced transport of GLUT4 vesicles to the plasma membrane. Elevated ROS activates protein kinase-D1 (PKD1) in a death-activated kinase (DAPK)-dependent manner, followed by activation of an unknown budding-inducing lipid or protein kinase (LK/PK), which enables the budding and fission of GLUT4 vesicles from the endosomal compartment. PKD1 and AMPK pathways regulate different processes that act in concert to ensure efficient contraction-induced GLUT4 translocation to the membrane and stimulation of glucose uptake. The constitutively active state of LKB1 and DAPK is indicated by a cycling arrow. (B) Insulin-induced GLUT4 translocation in myocytes. In both cardiac and skeletal myocytes, insulin activates two different insulin receptor (IR) pools. One pool colocalizes with caveolinorganized membrane rafts, and leads to activation of the insulin receptor substrate-1/2 (IRS1/2)/phosphoatidylinositol-3 kinase (PI3K)/Akt pathway, resulting in TBC1D4 phosphorylation. The other pool colocalizes with flotillinorganized microdomains, leading to activation of the $\mathrm{Cbl} / \mathrm{C} 3 \mathrm{G} / \mathrm{TC} 10$ pathway. This pathway is activated by insulin in skeletal muscle as well as in heart [51,52]. TC10 is a member of the Rho family of small GTP-binding proteins, and is involved in speculate that at least one of these proteins might be activated by PKD1 to initiate the fission of GLUT4-containing vesicles upon the onset of contraction.

\section{Dual signaling input controls GLUT4 translocation}

Based on the findings discussed above, we may speculate that, at least in heart, AMPK and PKD1 are each required for contraction-induced GLUT4 translocation. Hence, AMPK activation will not lead to GLUT4 translocation if the PKD1 pathway is not operative. Conversely, PKD1 activation will not result in GLUT4 translocation if the AMPK pathway is not operative. Then, the question arises how the two kinases are positioned relative to each other. Several lines of evidence indicate that AMPK and PKD1 function via separate pathways. In cell-free systems, fulllength constitutively active $\mathrm{PKD} 1$ does not activate recombinant AMPK and vice versa [35]. In cardiomyocytes, when either PKD1 or AMPK $\alpha 2$ are silenced by small interfering (si)-RNA methodology or by cardio-specific gene KO (PKD1 $\mathrm{KO}$ mice and $\mathrm{AMPK} \alpha 2 \mathrm{KO}$ mice), the other kinase is normally activated by contraction [34,35]. This suggests the existence of two separate contraction-activated pathways for GLUT4 translocation, one involving AMPK and its upstream activating kinase LKB1 (or perhaps CaMKK $\beta / \mathrm{TAK} 1$ as an alternative upstream kinase), and the other involving PKD1 and its upstream kinase DAPK. The simultaneous activation of both pathways appears to be mandatory for contraction-induced glucose uptake. Indeed, cardiac contractile activity through enhanced ATP utilization and oxidative phosphorylation may result in the concomitant formation of AMP and ROS, which are second messengers triggering activation of AMPK and PKD1, respectively (Figure 1A). The dual signaling input concept, as delineated in vitro upon inducing contractions in quiescent cardiomyocytes in culture via electric field stimulation $[34,35]$, is expected to also be of relevance in vivo in the continuously contracting heart, given the ability of the human heart to increase its beating rate by up to threefold during intensive exercise (Box 1).

Notably, the LKB1-AMPK and DAPK-PKD1 pathways share several common principles. For example, each of the signaling branches in contraction-induced GLUT4 translocation involves LKB1 and DAPK, upstream kinases that function as tumor suppressor proteins and exhibit constitutive activity (at least under some circumstances). In agreement with this notion, the increased phosphorylation of AMPK and PKD1 upon contraction stimulation may rather be a consequence of conformational changes within these kinases induced by their second messengers AMP and ROS, respectively, thereby allowing the tumor suppressors to gain access to their respective substrates. PKD1 and AMPK also each exert other direct functions in contraction. AMPK has been reported to phosphorylate components of the contractile apparatus, such as the smooth muscle myosin light chain kinase [44], and regulates the myosin regulatory light chain through an indirect

the regulation of actin remodeling. Actin remodeling creates a cytoskeleta fibrous network that enables GLUT4 vesicles to travel from endosomes to the membrane. TC10 also binds to Exo70, a protein constituent of the GLUT4 fission complex. 
Box 1. In vivo implication of the dual input stimulation of GLUT4 translocation in the heart

The dual signaling input concept of contraction-induced GLUT4 translocation was delineated from the observation in primary cardiomyocyte cultures that knockdown and/or KO of either AMPK or PKD1 fully blocked 4-Hz contraction-induced GLUT4 translocation [34]. In the absence of contractions, these cardiomyocytes in vitro display low rates of glucose uptake. This basal glucose uptake is mediated largely by the non-inducible glucose transporter GLUT1, and also by low basal surface levels of GLUT4 [3]. AMPK or PKD1 deletion had no effect on these low basal rates [34]. Hence, the dual signaling input requirement does not contribute significantly to glucose uptake under conditions with minimal energetic demands. By contrast, in the in vivo situation, the heart is continuously contracting (in humans at a rate of $60-100$ beats $/ \mathrm{min}$ ) when at rest. Under resting conditions, AMPK and PKD1 would be expected to display a 'basal activation level' and a concomitant minimal contribution of the dual input signaling to GLUT4 translocation to the basal in vivo glucose uptake rate. Depending on age and fitness, the resting heart rate in humans can be increased approximately threefold during intensive exercise [63]. In rats, the increase in heart rate due to a gradual increase in treadmill exercise has been shown to be accompanied by a gradual increase in AMPK activity up to fourfold depending on the exercise intensity, and a proportional increase in GLUT4 translocation [64]. Although these in vivo studies did not include investigation of PKD1 activation, gradual PKD1 activation up to fivefold in cardiomyocytes in vitro has been demonstrated, depending on the applied electrical stimulation frequency [50]. Likely, the dual input signaling input for contraction-induced GLUT4 translocation, as delineated in vitro, is of similar importance in vivo.

mechanism [45]. Limited knowledge is available for cardiac muscle, except that AMPK phosphorylates cardiac-troponin-I (cTnI) at Ser150 to increase myofilament $\mathrm{Ca}^{2+}$ sensitivity $[46,47]$. PKD phosphorylates several contractile proteins, including $\mathrm{cTnI}$ and cardiac myosin-binding protein C (c-MyBPc) $[48,49]$. In particular, the ability of PKD to phosphorylate cTnI at Ser23/24 implicates a role of PKD in the acceleration of relaxation through increased myofilament $\mathrm{Ca}^{2+}$ sensitivity and crossbridge cycling [49]. Moreover, contraction stimulation has been shown to lead to cMyBPc phosphorylation in a PKD1-dependent manner, where phosphorylation serves to increase the maximal tension [50]. When integrating the phosphorylation of myofilament proteins mediated by both AMPK and PKD1 with their critical roles in GLUT4 translocation, the notion arises that both key kinases have broad involvement in the responses of the heart to contraction. In this way, AMPK and PKD1 could connect the changes in energy demand resulting from a change (not only acceleration, but also likely in case of a deceleration) of contraction mechanics to a concomitant change (increase or decrease, respectively) in uptake of energy-providing substrates, especially glucose. Taken together, the LKB1-AMPK and DAPKPKD1 cascades may have evolved towards similar functions, because the vital processes that they regulate required a robust signaling module with direct input from second messengers of contraction signaling.

The dual signaling concept that we propose may not be uniquely restricted to cardiac contraction-induced GLUT4 translocation, because there is evidence that insulin-induced GLUT4 translocation in skeletal muscle cells (and adipocytes) [51-54], as well as in heart [51], is mediated by two independent pathways (Figure 1B). Namely, insulin stimulates PKB/Akt-dependent TBC1D4 phosphorylation and a parallel pathway compartmentalized in plasma membrane lipid rafts involving the proto-oncogene $\mathrm{Cbl}$, the guanine nucleotide exchange factor C3G, and a Rhofamily small GTP-binding protein TC10. The similarities in dual signaling input regulation of insulin-induced GLUT4 translocation and contraction-induced GLUT4 translocation are apparent. Namely, there is a common signaling branch landing on TBC1 family members (insulin-induced GLUT4 translocation, via PKB/Akt; contraction-induced GLUT4 translocation, via AMPK), whereas the other branch might activate components of the vesicle fission machinery (insulin-induced GLUT4 translocation, via TC10; contraction-induced GLUT4 translocation, via one of the three proposed kinases functioning in vesicle budding, most likely PI4KB [32]). The physiological function of this dual signaling input may be to serve as a control mechanism to ensure that GLUT4 translocation will be initiated under specific metabolic conditions only (i.e., insulin and contraction). The dual input concept would then provide an explanation for the inability of the frequently used AMPK activators AICAR or A-7692766 to stimulate glucose uptake into cardiomyocytes [16,34]. By contrast, another compound commonly used to activate AMPK (i.e., the mitochondrial $\mathrm{F}_{1} \mathrm{~F}_{0}$-ATPase inhibitor oligomycin) successfully stimulates both GLUT4 translocation and glucose uptake $[34,35]$. The explanation for these apparent paradoxical observations is that oligomycin additionally stimulates PKD1 activation (in a ROS-dependent manner), while AICAR does not [34].

Taken together, efficient control of cellular GLUT4 translocation putatively requires more than a single input signal. Preliminary evidence suggests that Ras-related C3 botulinum toxin substrate-1 (Rac1) participates in both insulin [55] and contraction-induced [56] GLUT4 translocation to organize the cortical actin network through polymerization-depolymerization cycles into a mobile highway for traveling GLUT4 vesicles. In contraction-induced GLUT4 translocation, this Rac1 involvement is independent of AMPK [56]. Thus, Rac1 might be a component of a separate signaling axis, but further corroboration is required.

\section{Pathophysiological implications of the dual input requirement}

The diabetic heart is characterized by an increase in left ventricular mass in combination with diastolic dysfunction [57]. This maladaptive remodeling and impairment in contractile function has been associated with altered substrate preference. Whereas in the normal heart, glucose and long-chain fatty acids are the main energy substrates and contribute about equally to ATP production for cardiac contraction, the diabetic heart shifts to fatty acids at the expense of glucose [58]. In fact, the increase in fatty acid uptake is a key early event in the development of diabetic cardiomyopathy $[58,59]$, subsequently leading to accumulation of bioactive lipid species (DAG and ceramides), which then impair insulin signaling at the level of insulin-receptor substrate-1/2 (DAG) and Akt (ceramides) [1]. Consequently, insulin-stimulated glucose uptake is impaired in the diabetic heart. 
Strategies to increase glucose uptake into the diabetic heart would be beneficial, because they would alleviate the insulin-resistant state of the heart and thereby reverse the maladaptive remodeling process associated with insulin resistance. A desired strategy to increase glucose uptake in the diabetic heart might be via increased contraction-induced GLUT4 translocation, because contraction stimulation recruits GLUT4 to the sarcolemma independently of the insulin-signaling axis [6,7].

According to the dual signaling input hypothesis, both AMPK (to stimulate GLUT4 vesicular trafficking) and PKD1 (to enhance endosomal GLUT4 vesicle budding) need to be activated. However, when the activation state of one is higher than that of the other, activation of the latter may suffice. This might explain why cardio-specific overexpression of constitutively active PKD1 in mice results in increased cardiac glucose uptake [37]. Namely, the PKD1-mediated rise in glucose uptake would only be possible when the resident AMPK activity in the hearts of these cardio-specific PKD1 overexpressor mice is sufficiently high. The elevated AMPK activation state in this particular case may be due to the continuous cardiac contractile activity in the in vivo setting [26]. Assuming that AMPK activity is ample in the beating heart, one could argue that PKD1 is the preferable target kinase to increase glucose uptake into the diabetic heart. Proof-of-principle for PKD1 targeting as a promising antidiabetic strategy has been obtained by the observation that cardio-specific overexpression of constitutively active PKD1 in mice fed a diabetogenic Western diet prevented the onset of insulin resistance in the heart, as well as the development of maladaptive changes in cardiac morphology [37]. However, it should be taken into account that mice overexpressing a cardio-specific constitutively active PKD1 construct develop cardiac hypertrophy early in life and display greatly increased mortality [37,60]. Furthermore, because PKD1 is involved in multiple cellular processes, its stimulation will alter these processes in addition to upregulating glucose import. Nevertheless, the PKD1 pathway offers therapeutic opportunities that require exploration, possibly as a short-term treatment (to avoid hypertrophic growth) or focusing on the downstream signaling events, such as GLUT4 vesicle budding (to circumvent the broad cellular effects).

\section{Concluding remarks}

Further research is needed to decipher the signaling processes downstream of PKD1 leading to GLUT4 translocation. Given that the dual signaling input requirement originates from studies in cardiac muscle, the presence of two independent pathways for contraction-induced GLUT4 translocation in skeletal muscle should also be investigated. Possible differences between heart and muscle were highlighted by the observation that AICAR effectively increases glucose uptake into muscle $[9,61]$, as opposed to heart. This could be due to the low basal level of resident AMPK activity in resting muscle (compared with the contracting heart), rendering AMPK in this tissue as a more important control site for dual input regulation of GLUT4 translocation. Thus, in absence of additional information on the role of PKD1 in muscle and due to safety considerations, AMPK remains the more eligible kinase to target to upregulate GLUT4 translocation for the prevention and/or treatment of insulin resistance in skeletal muscle.

\section{Acknowledgment}

D.N. is the recipient of a VIDI-Innovational Research Grant from the Netherlands Organization of Scientific Research (NWO-ALW Grant no. 864.10.007).

\section{References}

1 Savage, D.B. et al. (2007) Disordered lipid metabolism and the pathogenesis of insulin resistance. Physiol. Rev. 87, 507-520

2 Till, M. et al. (1997) Molecular mechanisms of contraction-induced translocation of GLUT4 in isolated cardiomyocytes. Am. J. Cardiol. $80,85 \mathrm{~A}-89 \mathrm{~A}$

3 Abel, E.D. (2004) Glucose transport in the heart. Front. Biosci. 9, 201215

4 Zorzano, A. et al. (1997) Regulation of glucose transport, and glucose transporters expression and trafficking in the heart: studies in cardiac myocytes. Am. J. Cardiol. 80, 65A-76A

5 Morgan, B.J. et al. (2011) GLUT4 associated proteins as therapeutic targets for diabetes. Recent Pat. Endocr. Metab. Immune Drug Discov. $5,25-32$

6 Luiken, J.J. et al. (2003) Contraction-induced fatty acid translocase/ CD36 translocation in rat cardiac myocytes is mediated through AMPactivated protein kinase signaling. Diabetes 52, 1627-1634

7 Lund, S. et al. (1995) Contraction stimulates translocation of glucose transporter GLUT4 in skeletal muscle through a mechanism distinct from that of insulin. Proc. Natl. Acad. Sci. U.S.A. 92, 5817-5821

8 Viollet, B. et al. (2007) Targeting AMP-activated protein kinase as a novel therapeutic approach for the treatment of metabolic disorders. Diabetes Metab. 33, 395-402

9 Kurth-Kraczek, E.J. et al. (1999) 5' AMP-activated protein kinase activation causes GLUT4 translocation in skeletal muscle. Diabetes 48, 1667-1671

10 Hardie, D.G. (2011) Energy sensing by the AMP-activated protein kinase and its effects on muscle metabolism. Proc. Nutr. Soc. 70, 92-99

11 Richter, E.A. et al. (2013) Exercise, GLUT4, and skeletal muscle glucose uptake. Physiol. Rev. 93, 993-1017

12 Jorgensen, S.B. et al. (2004) Knockout of the alpha2 but not alpha1 5'AMP-activated protein kinase isoform abolishes 5-aminoimidazole-4carboxamide-1-beta-4-ribofuranosidebut not contraction-induced glucose uptake in skeletal muscle. J. Biol. Chem. 279, 1070-1079

13 Habets, D.D. et al. (2009) Crucial role for LKB1 to AMPKalpha2 axis in the regulation of CD36-mediated long-chain fatty acid uptake into cardiomyocytes. Biochim. Biophys. Acta 1791, 212-219

14 Sakamoto, K. et al. (2005) Deficiency of LKB1 in skeletal muscle prevents AMPK activation and glucose uptake during contraction. EMBO J. 24, 1810-1820

15 Sakamoto, K. et al. (2006) Deficiency of LKB1 in heart prevents ischemia-mediated activation of AMPKalpha2 but not AMPKalpha1. Am. J. Physiol. Endocrinol. Metab. 290, E780-E788

16 Angin, Y. et al. (2014) Calcium signaling recruits substrate transporters GLUT4 and CD36 to the sarcolemma without increasing cardiac substrate uptake. Am. J. Physiol. Endocrinol. Metab. 307, E225-E236

17 Alessi, D.R. et al. (2006) LKB1-dependent signaling pathways. Annu. Rev. Biochem. 75, 137-163

18 Neumann, D. et al. (2007) Co-expression of LKB1, MO25alpha and STRADalpha in bacteria yield the functional and active heterotrimeric complex. Mol. Biotechnol. 36, 220-231

19 Cartee, G.D. (2015) Roles of TBC1D1 and TBC1D4 in insulin- and exercise-stimulated glucose transport of skeletal muscle. Diabetologia $58,19-30$

20 Chen, S. et al. (2011) Mice with AS160/TBC1D4-Thr649Ala knockin mutation are glucose intolerant with reduced insulin sensitivity and altered GLUT4 trafficking. Cell Metab. 13, 68-79

21 Peck, G.R. et al. (2006) Interaction of the Akt substrate, AS160, with the glucose transporter 4 vesicle marker protein, insulin-regulated aminopeptidase. Mol. Endocrinol. 20, 2576-2583 
22 Ramm, G. et al. (2006) A role for 14-3-3 in insulin-stimulated GLUT4 translocation through its interaction with the RabGAP AS160. J. Biol. Chem. 281, 29174-29180

23 Kramer, H.F. et al. (2006) AS160 regulates insulin- and contractionstimulated glucose uptake in mouse skeletal muscle. J. Biol. Chem. 281, 31478-31485

24 Ducommun, S. et al. (2012) Thr649Ala-AS160 knock-in mutation does not impair contraction/AICAR-induced glucose transport in mouse muscle. Am. J. Physiol. Endocrinol. Metab. 302, E1036-E1043

25 Taylor, E.B. et al. (2008) Discovery of TBC1D1 as an insulin-, AICAR-, and contraction-stimulated signaling nexus in mouse skeletal muscle. J. Biol. Chem. 283, 9787-9796

26 Lee, M.T. et al. (2009) Structural mechanisms for regulation of membrane traffic by rab GTPases. Traffic 10, 1377-1389

27 Fukuda, M. (2011) TBC proteins: GAPs for mammalian small GTPase Rab? Biosci. Rep. 31, 159-168

28 Ishikura, S. et al. (2008) Muscle cells engage Rab8A and myosin Vb in insulin-dependent GLUT4 translocation. Am. J. Physiol. Cell Physiol. 295, C1016-C1025

29 Sadacca, L.A. et al. (2013) Specialized sorting of GLUT4 and its recruitment to the cell surface are independently regulated by distinct Rabs. Mol. Biol. Cell 24, 2544-2557

30 Cleland, P.J. et al. (1989) Exercise-induced translocation of protein kinase $\mathrm{C}$ and production of diacylglycerol and phosphatidic acid in rat skeletal muscle in vivo. Relationship to changes in glucose transport. $J$. Biol. Chem. 264, 17704-17711

31 Rozengurt, E. (2011) Protein kinase D signaling: multiple biological functions in health and disease. Physiology (Bethesda) 26, 23-33

32 Hausser, A. et al. (2005) Protein kinase D regulates vesicular transport by phosphorylating and activating phosphatidylinositol-4 kinase IIIbeta at the Golgi complex. Nat. Cell Biol. 7, 880-886

33 Storz, P. (2007) Mitochondrial ROS: radical detoxification, mediated by protein kinase D. Trends Cell Biol. 17, 13-18

34 Dirkx, E. et al. (2012) Protein kinase D1 is essential for contractioninduced glucose uptake but is not involved in fatty acid uptake into cardiomyocytes. J. Biol. Chem. 287, 5871-5881

35 Luiken, J.J. et al. (2008) Identification of protein kinase D as a novel contraction-activated kinase linked to GLUT4-mediated glucose uptake, independent of AMPK. Cell. Signal. 20, 543-556

36 Steinbusch, L.K. et al. (2013) Overexpression of AMP-activated protein kinase or protein kinase D prevents lipid-induced insulin resistance in cardiomyocytes. J. Mol. Cell. Cardiol. 55, 165-173

37 Dirkx, E. et al. (2014) Protein kinase-D1 overexpression prevents lipidinduced cardiac insulin resistance. J. Mol. Cell. Cardiol. 76, 208-217

38 Gruenberg, J. et al. (1995) Membrane transport in the endocytic pathway. Curr. Opin. Cell Biol. 7, 552-563

39 Dirkx, E. et al. (2013) Detection of cardiac myosin binding protein-C (cMyBP-C) by a phospho-specific PKD antibody in contracting rat cardiomyocytes. Adv. Biosci. Biotechnol. 4, 1-6

40 Eisenberg-Lerner, A. et al. (2007) DAP kinase regulates JNK signaling by binding and activating protein kinase $\mathrm{D}$ under oxidative stress. Cell Death Differ. 14, 1908-1915

41 Eisenberg-Lerner, A. et al. (2012) PKD is a kinase of Vps34 that mediates ROS-induced autophagy downstream of DAPk. Cell Death Differ. 19, 788-797

42 Ghanekar, Y. et al. (2005) Signalling for secretion. Nat. Cell Biol. 7, 851-853

43 Valente, C. et al. (2005) Purification and functional properties of the membrane fissioning protein CtBP3/BARS. Methods Enzymol. 404, 296-316
44 Horman, S. et al. (2008) AMP-activated protein kinase phosphorylates and desensitizes smooth muscle myosin light chain kinase. J. Biol. Chem. 283, 18505-18512

45 Banko, M.R. et al. (2011) Chemical genetic screen for AMPKalpha2 substrates uncovers a network of proteins involved in mitosis. Mol. Cell $44,878-892$

46 Chen, S. et al. (2014) Alpha1 catalytic subunit of AMPK modulates contractile function of cardiomyocytes through phosphorylation of troponin I. Life Sci. 98, 75-82

47 Nixon, B.R. et al. (2012) AMP-activated protein kinase phosphorylates cardiac troponin I at Ser-150 to increase myofilament calcium sensitivity and blunt PKA-dependent function. J. Biol. Chem. 287, 19136-19147

48 Bardswell, S.C. et al. (2010) Distinct sarcomeric substrates are responsible for protein kinase D-mediated regulation of cardiac myofilament $\mathrm{Ca}^{2+}$ sensitivity and cross-bridge cycling. J. Biol. Chem. 285, 5674-5682

49 Haworth, R.S. et al. (2004) Protein kinase D is a novel mediator of cardiac troponin I phosphorylation and regulates myofilament function. Circ. Res. 95, 1091-1099

50 Dirkx, E. et al. (2012) Protein kinase D increases maximal $\mathrm{Ca}^{2+}$ activated tension of cardiomyocyte contraction by phosphorylation of cMyBP-C-Ser315. Am. J. Physiol. Heart Circ. Physiol. 303, H323-H331

51 Gupte, A. et al. (2006) Activation of the Cbl insulin signaling pathway in cardiac muscle; dysregulation in obesity and diabetes. Biochem. Biophys. Res. Commun. 342, 751-757

52 Kanzaki, M. (2006) Insulin receptor signals regulating GLUT4 translocation and actin dynamics. Endocr. J. 53, 267-293

53 Saltiel, A.R. et al. (2003) Insulin signaling in microdomains of the plasma membrane. Traffic 4, 711-716

54 Zhou, Q.L. et al. (2004) Analysis of insulin signalling by RNAi-based gene silencing. Biochem. Soc. Trans. 32, 817-821

55 Sylow, L. et al. (2013) Rac1 signaling is required for insulin-stimulated glucose uptake and is dysregulated in insulin-resistant murine and human skeletal muscle. Diabetes 62, 1865-1875

56 Sylow, L. et al. (2015) Stretch-stimulated glucose transport in skeletal muscle is regulated by Rac1. J. Physiol. 593, 645-656

57 Boudina, S. et al. (2010) Diabetic cardiomyopathy, causes and effects. Rev. Endocr. Metab. Disord. 11, 31-39

58 Harmancey, R. et al. (2008) The complexities of diabetic cardiomyopathy: lessons from patients and animal models. Curr. Diab. Rep. 8, 243-248

59 Dirkx, E. et al. (2011) High fat diet induced diabetic cardiomyopathy. Prostaglandins Leukot. Essent. Fatty Acids 85, 219-225

60 Harrison, B.C. et al. (2006) Regulation of cardiac stress signaling by protein kinase d1. Mol. Cell. Biol. 26, 3875-3888

61 Merrill, G.F. et al. (1997) AICA riboside increases AMP-activated protein kinase, fatty acid oxidation, and glucose uptake in rat muscle. Am. J. Physiol. 273, E1107-E1112

62 Abbott, M.J. et al. (2009) CaMKK is an upstream signal of AMPactivated protein kinase in regulation of substrate metabolism in contracting skeletal muscle. Am. J. Physiol. Regul. Integr. Comp. Physiol. 297, R1724-R1732

63 Mahon, A.D. et al. (2010) Evaluating the prediction of maximal heart rate in children and adolescents. Res. Q. Exerc. Sport 81, 466-471

64 Coven, D.L. et al. (2003) Physiological role of AMP-activated protein kinase in the heart: graded activation during exercise. Am. J. Physiol. Endocrinol. Metab. 285, E629-E636 